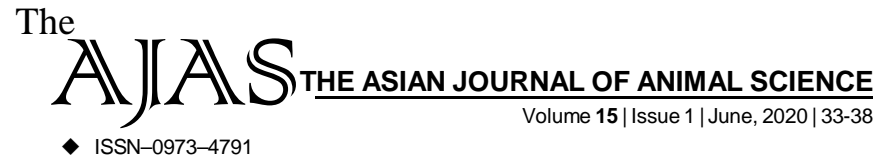

DOI : 10.15740/HAS/TAJAS/15.1/33-38 Visit us | www.researchjournal.co.in

A REVIEW

\title{
Antimicrobial resistance: A global public health and economic concern
}

Chandra Shekhar

Author for Corresponding -

\section{Chandra Shekhar}

Department of Veterinary Public

Health and Epidemiology,

College of Veterinary Science and

Animal Husbandry, Acharya

Narendra Deva University of

Agriculture and Technology,

Kumarganj, Ayodhya (U.P.)

India

Email: cshekharvph@gmail.com
ABSTRACT...... Antibiotic resistance is a global concern that is present in every country. Infections caused by drug-resistant bacteria increases the risk of clinical outcomes and death of patients and consume more health-care resources.Antimicrobial resistance (AMR) increases the cost of health care, long time period stays in hospitals and requirement of more intensive care. The success of major surgery and cancer chemotherapy would be compromised without effective antimicrobials. The cost of health care for patients with resistant infections is higher due to longer duration of illness, additional tests and use of more expensive drugs. AMR occurs naturally over time, usually through genetic changes. However, the misuse and overuse of antimicrobials accelerates this process. In many places, antibiotics are overused and misused in animals and humans and often given without professional supervision. Other factors that encourage the spread of antimicrobial resistance include inadequate sanitary conditions, inappropriate food-handling and poor infection control. Antimicrobial resistance (AMR) including multidrug resistance (MDR), is on the rise among many micro-organisms in healthcare facilities as well as in community. Antimicrobial resistance in infections caused by Escherichia coli, Klebsiellapneumoniae and Methicillin-resistant Staphylococcus aureus (MRSA) has posed a significant impact on health and economic burden across the world. AMR is an increasingly serious threat to global public health that requires action across all government sectors and society. The measures can limit the development and spread of antimicrobial resistance include hygiene and sanitation, vaccination, sustainable animal husbandry practices, health education, epidemiological surveillance and research, national action plan, optimization of use of antimicrobials, development of the economic case for sustainable investment, judicious use of antimicrobials and co-operation of international organizations/agencies.

KEY WORDS...... Antimicrobial, Resistance, Impact, Prevention, Control

HOW TO CITE THIS ARTICLE - Shekhar, Chandra (2020). Antimicrobial resistance: A global public health and economic concern. Asian J. Animal Sci., 15(1): 33-38. DOI:10.15740/HAS/TAJAS/15.1/ 33-38.Copyright@2020:HindAgri-Horticultural Society.

ARTICLE CHRONICLE - Received : 26.02.2020; Accepted : 25.05.2020 